\title{
The Use of a Standardized PCT-Algorithm Reduces Costs in Intensive Care in Septic Patients - A DRG-Based Simulation Model
}

\author{
M. H. Wilke ${ }^{1}$, R. F. Grube ${ }^{1}$, K. F. Bodmann ${ }^{2}$ \\ ${ }^{1}$ Dr. Wilke GmbH - inspiring.health, Munich, Germany \\ ${ }^{2} \mathrm{Head}$ of Internal Intensive Care Unit and Interdisciplinary Emergency Medicine Department, Klinikum Barnim - \\ Werner Forssmann Hospital, Eberswalde, Germany
}

\begin{abstract}
Introduction: The management of bloodstream infections especially sepsis is a difficult task. An optimal antibiotic therapy (ABX) is paramount for success. Procalcitonin (PCT) is a well investigated biomarker that allows close monitoring of the infection and management of ABX. It has proven to be a cost-efficient diagnostic tool. In Diagnoses Related Groups (DRG) based reimbursement systems, hospitals get only a fixed amount of money for certain treatments. Thus it's very important to obtain an optimal balance of clinical treatment and resource consumption namely the length of stay in hospital and especially in the Intensive Care Unit (ICU). We investigated which economic effects an optimized PCT-based algorithm for antibiotic management could have.
\end{abstract}

Materials and Methods: We collected inpatient episode data from 16 hospitals. These data contain administrative and clinical information such as length of stay, days in the ICU or diagnoses and procedures. From various RCTs and reviews there are different algorithms for the use of PCT to manage ABX published. Moreover RCTs and meta-analyses have proven possible savings in days of ABX (ABD) and length of stay in ICU (ICUD). As the meta-analyses use studies on different patient populations (pneumonia, sepsis, other bacterial infections), we undertook a short meta-analyses of 6 relevant studies investigating in sepsis or ventilator associated pneumonia (VAP). From this analyses we obtained savings in ABD and ICUD by calculating the weighted mean differences. Then we designed a new PCT-based algorithm using results from two very recent reviews. The algorithm contains evidence from several studies. From the patient data we calculated cost estimates using German National standard costing information for the German G-DRG system.

We developed a simulation model where the possible savings and the extra costs for (in average) 8 PCT tests due to our algorithm were brought into equation.

Results: We calculated ABD savings of -4 days and ICUD reductions of -1.8 days. Our algorithm contains recommendations for $\mathrm{ABX}$ onset (PCT $\geq 0.5 \mathrm{ng} / \mathrm{ml}$ ), validation whether ABX is appropriate or not (Delta from day 2 to day $3 \geq 30 \%$ indicates inappropriate $\mathrm{ABX})$ and recommendations for discontinuing $\mathrm{ABX}$ $(\mathrm{PCT} \leq 0.25 \mathrm{ng} / \mathrm{ml}$ ).
We received 278,264 episode datasets where we identified by computer-based selection 3,263 cases with sepsis. After excluding cases with length of stay (LOS) too short to achieve the intended savings, we ended with 1,312 cases with ICUD and 268 cases without ICUD. Average length of stay of ICU-patients was 27.7 \pm 25.7 days and for Non-ICU patients $17.5 \pm 14.6$ days respectively. ICU patients had an average of $8.8 \pm 8.7$ ICUD.

After applying the simulation model on this population we calculated possible savings of $€-1,163,000$ for ICU-patients and $€-36,512$ for Non-ICU patients.

Discussion: Our findings concerning the savings from the reduction of ABD are consistent with other publications. Savings ICUD had never been economically evaluated so far. Our algorithm is able to possibly set a new standard in PCT-based ABX. However the findings are based on data modelling. The algorithm will be implemented in 5-10 hospitals in 2012 and effects in clinical reality measured 6 months after implementation.

Conclusion: Managing sepsis with daily monitoring of PCT using our refined algorithm is suitable to save substantial costs in hospitals. Implementation in clinical routine settings will show how much of the calculated effect will be achieved in reality.

\section{Abbreviations}

ABD Days of antibiotic treatment

ABX Antibiotic Therapy

ALOS Average length of stay in a given DRG, basis for determining whether a patient causes more costs than reimbursement

CW cost-weight of a given DRG, directly related to clinical and economical "severity" (resource consumption)

DRG Diagnoses related groups, systems to classify patients based on their resource consumptions

HMV Hours of mechanical ventilation

ICD International Classification of Diseases

ICD-10 ICD, Revision 10

ICU Intensive care unit

ICUD treatment days on an intensive care unit

LOS Length of stay in hospital

NLOS Length of stay on normal ward; 
PCT Procalcitonin laboratory test - a biomarker

PDX Primary diagnosis (in ICD-10)

RCT Randomized control trial

SDX Secondary diagnosis (in ICD-10)

VAP Ventilator-associated pneumonia

\section{INTRODUCTION}

Sepsis is a severe disease with poor clinical as economical outcomes and a high mortality [1-3]. Moreover sepsis treatment does often not adhere to practice recommendations [4].

PCT is a well proven test for detection of bacterial infections and to determine start and end of antibiotic therapy in various settings [5-9]. Using PCT-based algorithms to monitor and manage antibiotic treatment saves ABD and ICUD as well as cost for antibiotics (by the effect of allowing an early de-escalation strategy). This has been proven in PCT-related RCTs and verified in meta-analyses and reviews [7, 8, 10, 11].

The cost-efficiency of using PCT to reduce cost of antibiotic treatment was also shown $[12,13]$.

In Germany hospitals are reimbursed by Diagnoses Related Groups (DRG) determined payments. Due to the nature of DRG-based reimbursement systems clinical and economical optimization should focus on the reduction of LOS especially ICUD [14, 15]. In many hospitals cost-saving efforts are centered on single areas like medication cost, cutting staff expenses or other without calculating the effects on the complete treatment process.

However, so far no publications are available which investigate the economic effect of the possible savings by using PCT-based treatment algorithms in a DRGreimbursement setting.

We conducted a study to determine possible savings in medication costs and costs for ICU-treatment using DRG data and favourable effects of a PCT-based treatment algorithm in sepsis patients.

\section{Materials AND METHOds}

We retrieved patient level DRG routine data from 16 hospitals in Germany. All patients were treated in 2010. The German National minimal basic datasets (MDBS) contains episode data (admission and discharge date, ICUD) and diagnoses (one PDX and up to $50 \mathrm{SDX}$ ).

ICUD are reported if the patient is treated in a specialised intensive-care unit. From previous data analyses we know that there exists an amount of patients treated in intermediate care units.

We developed an algorithm to detect sepsis patients by using the various ICD-10 codes and examining PDX and SDX of all patients. Once a sepsis code occurred the patients were flagged as 'Sepsis present'. By using the ICUD provided from the hospitals we further determined whether the patient was treated on ICU or not. If so, we flagged the patients as 'ICU treated'. Patients being on intermediate care units or small intensive care units without ICUD being present in the dataset we flagged as 'Non-ICU'.

Existing reviews on PCT are often based on RCTs with varying sample structures without considering in- fluences of the different sample structures. Although favourable outcomes are proven, we decided to calculate possible positive effects of a PCT-based algorithm by pooling data from RCTs which mainly deal with sepsis patients or at least had sepsis patient or bloodstream infections as subgroups. As ICUD and ABD are continuous variables we calculated weighted means according to the recommendations in the QUOROM statement [16].

Using the results from a recent review on PCTbased algorithms for the management of infections [7], we calculated an average utilization of 7 subsequent PCT-tests. The average cost for a PCT-test in Germany is $€ 25$ (based on data from the vendor). This would reflect the application of an algorithm based on daily testing.

By using the German National costing manual, we estimated treatment costs for each patient as the hospitals did not deliver data from the German National Cost data collection. The Institute for the reimbursement system in hospitals - InEK provides national average costs for each German DRG. However these costs are strongly related to the LOS in hospitals and to the ICUD. Therefore we calculated costs for the patients by calculating the following cost proportions from the national average costs:

- ICU costs: the German average costs for one day of ICU treatment is app. $€ 1,100$ [3]. ICUD were multiplied with these costs

- LOS-related costs: Treatment on the regular ward (including intermediate care), the use of diagnostic departments and medication costs show a strong correlation to the LOS. We calculated a NLOS by subtracting the ICUD from the LOS. NLOS was multiplied with the LOS-related costs

- Therapy related costs: Each DRG contains costs for the main therapy in hospital like surgery or cardiologic interventions or anaesthesia. These costs were directly taken from the national cost averages for each DRG

Thus we were able to estimate patient individual costs dependent on the individual LOS and ICUD.

To calculate savings from applying the PCT-based algorithm on our populations we used the following assumptions:

- ICUD according to the pooled analyses can only be saved, if ICUD of the individual patient is at least one day longer than the estimated savings. Thus we avoided implausible results if patients had e.g. only one ICUD. As medication costs (including antibiotics) are already part of the cost calculation for one day of ICU treatment, we did not reduce medication costs in patients with ICUD present

- For all patients without ICUD present, we only calculated savings from shorter ABD. As data on intermediate care stays were not available, we chose this conservative approach in order to avoid overestimation of possible savings. Costs for antibiotic treatment in sepsis vary $[3,7,13]$. We chose to build a flexible range for these costs in our simulation model with average daily costs of $€ 66$ 
The simulation model was built by including all patients detected as 'sepsis' patients in the following (simplified) equation:

Cost (PCT-based algorithm) = original costs - costs respecting savings + costs for additional PCT-testing.

PCT-tests are already performed in most of the hospitals in this study but the utilization of the results in terms of making treatment decisions is low [4]. Therefore we decided not to take the current cost in account. This is - again - a conservative estimate, as hospitals that will start using an optimized treatment algorithm will most likely not produce costs of $7 \mathrm{ex}$ tra tests but only the incremental average increase.

\section{RESULTS}

We collected data from several RCTs to determine possible favourable effects of PCT [9, 17-21]. The studies had the following results as shown in Table 1.

We included 6 studies. From the Boudma publication of the PRORATA trial we extracted the results from sepsis patients as the trial contained several different infections. The publication from Stolz was based on ventilator-associated pneumonia. However a number of patients suffered from septic shock and/or bacteraemia. Moreover VAP is a severe bacterial infection on the ICU.

By pooling the results and calculated the weighted means as well as the weighted mean difference (WMD) of all trials we found the following results as basis for our simulation model (Table 2).

For the further calculation we used possible reduction in $\mathrm{ABD}$ of -4 days and a possible reduction in ICUD of -1.8 days.

The weighted mean for days of antibiotic therapy (ABD) was 7.3 in the pooled PCT-groups. Therefore we took the assumption that 8 PCT-test will be a realistic frequency. We developed an algorithm based on recent reviews and publications on PCT in sepsis pa- tients $[7,13]$. The findings from Charles et.al. strongly recommend daily PCT-testing as the Delta between day 2 and day 3 shows a significant correlation to adequate versus inadequate ABX [5]. Figure 1 shows our recommended algorithm.

We received 278,264 datasets. In 3,263 episodes sepsis was detected and the cases were included in our analysis. 2,968 cases had at least one day of ICU stay (Range: 1-32). 1,312 cases had at least 3 ICUD and were included in the calculation of the savings in the ICU. 268 out of 295 Non-ICU cases had a total LOS that was at least 6 days and therefore the possible savings of $4 \mathrm{ABD}$ were applied.

The Figure 2 gives an overview of the patient selection. Table 3 shows the overall financial results after applying the simulation model.

Introducing the suggested algorithm for PCT-based ABX saves $€ 1,199,512$ in our sample population. As ICU-costs are tremendous, the savings achieved by shortening ICUD outweigh the savings achieved through reduction of ABD. However, even ABD-reduction covers the extra-costs for daily PCT-testing. As most hospitals in our sample do already measure PCT but not following strict algorithms the savings are even higher because we assumed all 8 PCT-tests will cause extra cost.

In average $€ 886.4$ can be saved, when patients need intensive care and $€ 136.2$ can be saved per case when patients are Non-ICU.

\section{Discussion}

We found that our results about reduction of ABXcosts are consistent with the results in other studies. Heyland et.al. assumed a reduction of 2 ABD with avg. daily $\mathrm{ABX}$-costs of Can $\$ 148.26$ using a regimen of avg. 6 PCT-test which cost Can\$49.42 [13]. All other published results did not explicitly refer to ABX costs but to the reduction of ABD. A reduction of

Table 1.

\begin{tabular}{|c|c|c|c|c|c|c|c|c|}
\hline Author & Year & Patient population & $\begin{array}{l}\text { Cases } \\
\text { PCT }\end{array}$ & $\begin{array}{l}\text { Cases } \\
\text { Control }\end{array}$ & $\begin{array}{l}\text { ABD } \\
\text { PCT }\end{array}$ & $\begin{array}{l}\text { ABD } \\
\text { Control }\end{array}$ & $\begin{array}{l}\text { ICUD } \\
\text { PCT }\end{array}$ & $\begin{array}{l}\text { ICUD } \\
\text { Control }\end{array}$ \\
\hline Nobre et.al. & 2008 & Sepsis & 31 & 37 & 6 & 10 & 3 & 5 \\
\hline Hochreiter & 2009 & SIRS & 57 & 53 & 5.9 & 7.9 & 15.5 & 17.7 \\
\hline Bouadma & 2010 & Various, only Sepsis used! & 55 & 53 & 9.8 & 12.8 & n.a. & n.a. \\
\hline Svoboda & 2007 & postoperative Sepsis & 38 & 34 & n.a. & n.a. & 16.1 & 19.4 \\
\hline Stolz & 2009 & VAP (Sepsis included) & 51 & 50 & 7 & 15 & n.a. & n.a. \\
\hline Schroeder & 2009 & Surgical sepsis patients & 14 & 13 & 6.6 & 8.3 & n.a. & n.a. \\
\hline
\end{tabular}

Table 2.

\begin{tabular}{lcccc}
\hline & \multicolumn{2}{c}{ ABD (5 studies) } & \multicolumn{2}{c}{ ICUD (3 studies) } \\
& ABD PCT & ABD Control & ICUD PCT & ICUD Control \\
\hline $\begin{array}{l}\text { Weighted Mean (days) } \\
\text { WMD (days) }\end{array}$ & 7.3 & 11.3 & 12.6 & 14.4 \\
\hline
\end{tabular}




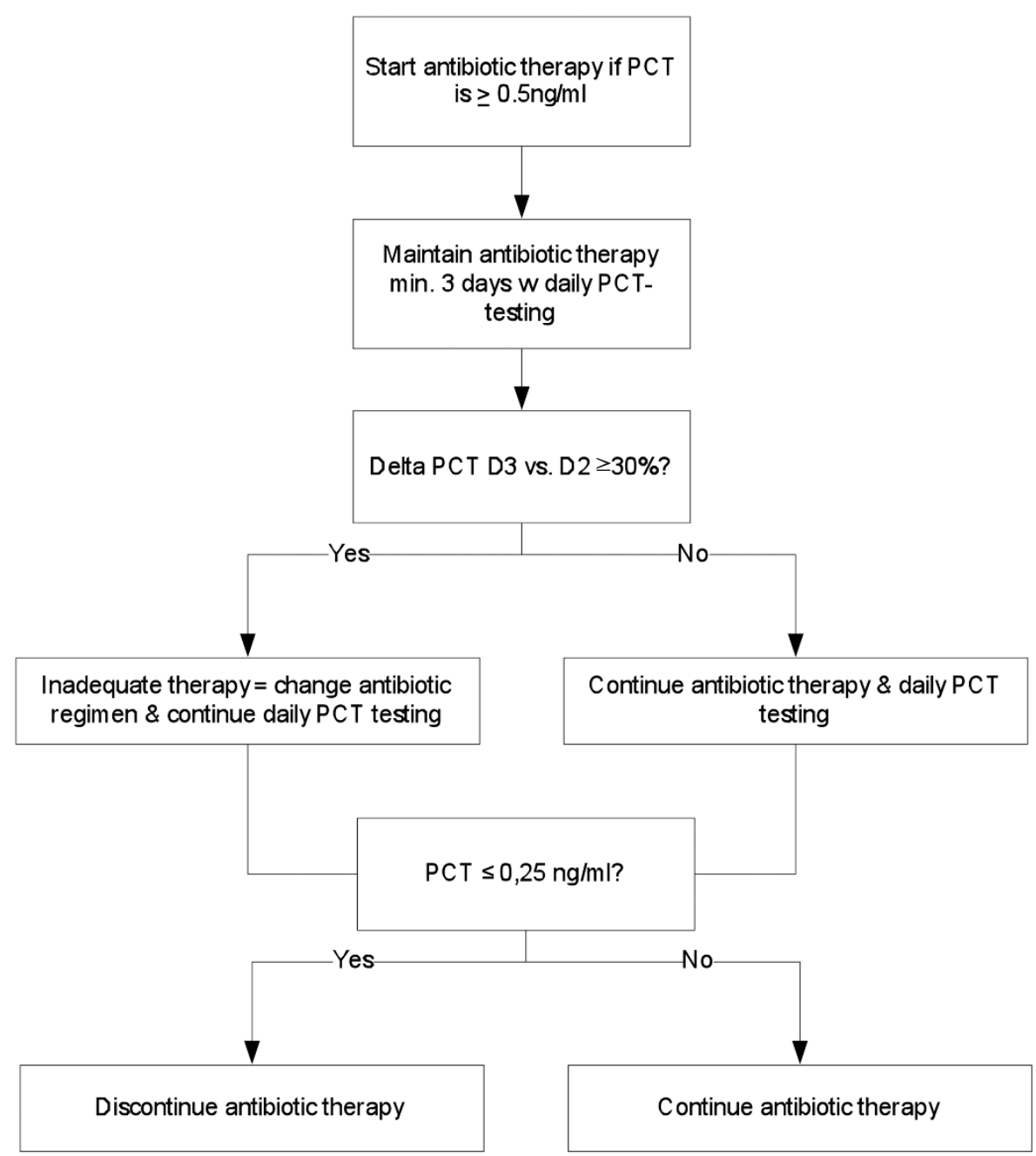

Fig. 1.

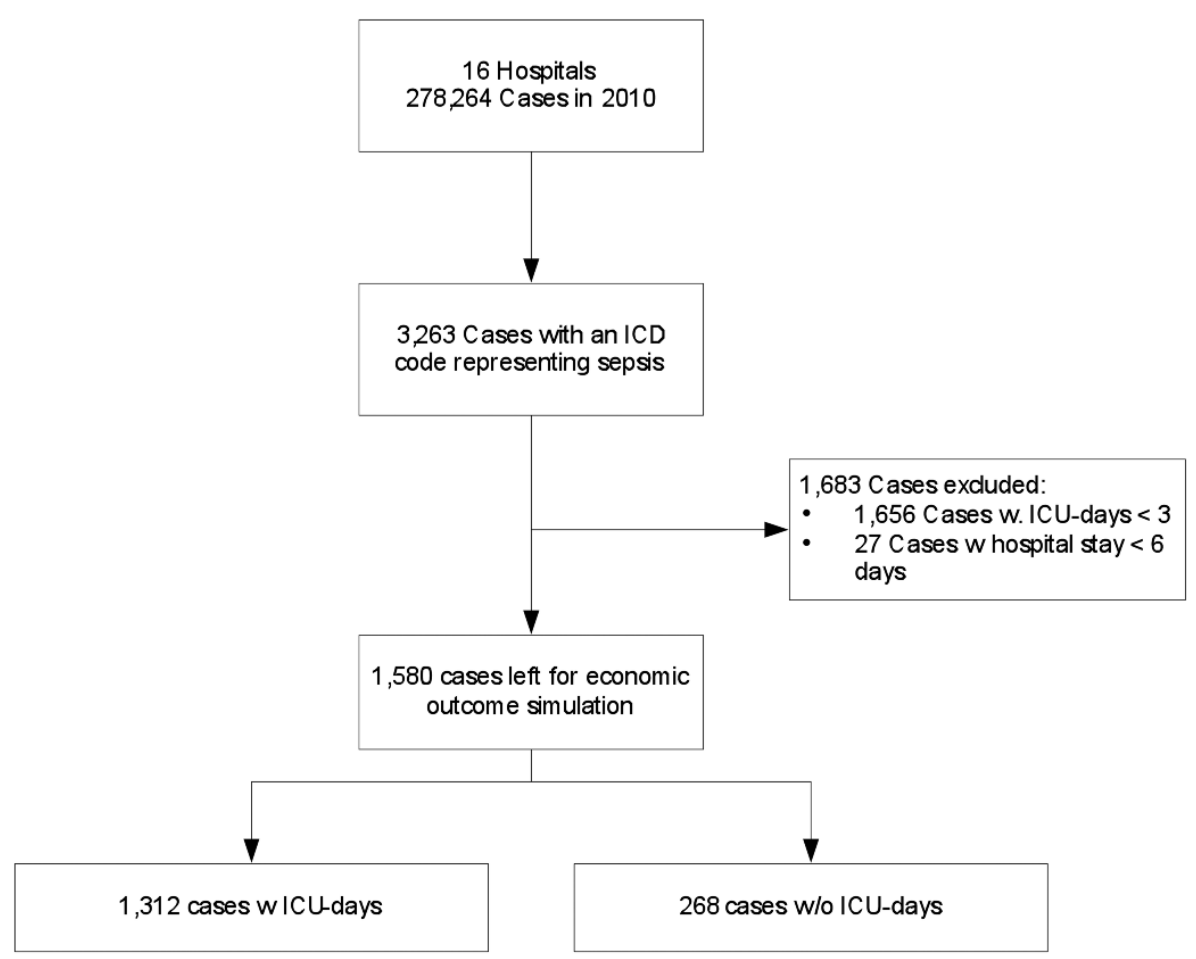

Fig. 2. 
Table 3.

\begin{tabular}{lcc}
\hline & ICU - Cases & Non-ICU Cases \\
\hline $\mathrm{n}=$ & 1,312 & 268 \\
ALOS & $27.7 \pm 25.7$ & $17.5 \pm 14.6$ \\
National ALOS & 22.7 & 10.3 \\
Avg. ICUD & $8.8 \pm 8.7$ & 0 \\
Costs before applying PCT-Algorithm & & $1,667,000$ \\
Total Costs $(€)$ & $24,700,000$ & 0 \\
ICU-Costs $(€)$ & $12,788,000$ & 266,499 \\
Medication Costs $(€)$ & $2,205,000$ & 110,000 \\
Laboratory Costs $(€)$ & $2,140,000$ & \\
Costs after applying PCT-Algorithm & & $1,630,488$ \\
Total Costs $(€)$ & $23,537,000$ & 0 \\
ICU-Costs $(€)$ & $11,314,000$ & 176,387 \\
Medication Costs $(€)$ & $1,877,000$ & 163,600 \\
Laboratory Costs $(€)$ & $2,381,000$ & $\mathbf{- 3 6 , 5 1 2}$ \\
Total Savings $(€)$ & $-\mathbf{1 , 1 6 3 , 0 0 0}$ & \\
\hline
\end{tabular}

ICUD most certainly leads to substantial savings, as the cost per day are much higher than the costs for PCT-testing. However in the real life a cost reduction may only occur in terms of variable costs, because if less ICUD are needed to treat sepsis patients when applying the PCT-based algorithm, the fixed costs in the ICU remain untouched. On the other hand in most German ICUs there's a constant shortage of available beds and various strategies are carried out to meet the demand [22-24]. Therefore the full daily ICU costs can be taken in account assuming that the bed is used by a new patient and a constant utilization of the ICU is maintained even if the optimized therapy strategies lead to shorter ICUD in sepsis.

The results shown in this study are based on predictive modelling. We already remarked that modelling is only part of the story [15]. From our point of view each model has to be proven in clinical reality. Knowing the obstacles as low adaption rate of practice guidelines [4], this is a challenging task. We plan to implement the algorithm described in this paper in 5-10 hospitals in 2012 and measure the effects on ICUD and $\mathrm{ABD}$ in the clinical reality.

\section{CONCLUSION}

By using a standardized algorithm for PCT-guided ABX-Therapy and assuming that the effects of ABD and ICUD derived from former studies will apply to the selected patient population the use of PCT is highly cost-effective!

$\mathrm{ABD}$ and ICUD reductions results in savings of up to $€ 1,199,512$ or $€ 886.4$ in average for ICU-patients and $€ 136.2$ for Non-ICU patients.

As shown in many other trials the clinical outcome is not negatively affected if $\mathrm{ABX}$ is shortened using PCT-based algorithms.
The model we developed will be used for further investigations. After implementation in clinical reality direct measurement of the effects will be possible.

Funding and possible bias

This study was supported by an unrestricted research grant from B.R.A.H.M.S GmbH, Henningsdorf, Germany. The funding party had no influence on the design of the algorithms and the economic modelling.

\section{Conflict of interest disclosures}

M. H. Wilke received consultancy fees and honoraria for lectures from Pfizer Pharma GmbH, Novartis Pharma Gmbh, and Wyeth Pharma GmbH (until 2009) and B.R.A.H.M.S $\mathrm{GmbH}$.

R. F. Grube received consultancy fees from Pfizer Pharma GmbH, Novartis Pharma Gmbh, and Wyeth Pharma GmbH (until 2009) and B.R.A.H.M.S GmbH.

K. F. Bodmann received honoraria for lectures from Pfizer Pharma GmbH, Novartis Pharma GmbH, Bayer Pharma $\mathrm{GmbH}$, Infectopharm GmbH, Janssen-Cilag $\mathrm{GmbH}$, B.R.A.H.M.S GmbH, MSD Merck, Sharp \& Dome GmbH

\section{REFERENCES}

1. Gastmeier P, Sohr D, Geffers C, Zuschneid I, Behnke M, Ruden H. [Mortality in German intensive care units: dying from or with a nosocomial infection?]. Anasthesiologie, Intensivmedizin, Notfallmedizin, Schmerztherapie : AINS 2005;40:267-72.

2. Brunkhorst FM. [Epidemiology, economy and practice -results of the German study on prevalence by the competence network sepsis (SepNet)]. Anasthesiologie, Intensivmedizin, Notfallmedizin, Schmerztherapie : AINS 2006;41:43-4.

3. Moerer O, Burchardi H. [The cost of sepsis]. Der Anaesthesist 2006;55 Suppl 1:36-42.

4. Brunkhorst FM, Engel C, Ragaller M, et al. Practice and perception--a nationwide survey of therapy habits in sepsis. Critical care medicine 2008;36:2719-25. 
5. Charles PE, Tinel C, Barbar S, et al. Procalcitonin kinetics within the first days of sepsis: relationship with the appropriateness of antibiotic therapy and the outcome. Critical care (London, England) 2009;13:R38.

6. Harbarth S, Albrich WC, Muller B. When once is not enough--further evidence of procalcitonin-guided antibiotic stewardship. Critical care (London, England) 2009;13:165.

7. Hochreiter M, Schroeder S. [Procalcitonin-based algorithm. Management of antibiotic therapy in critically ill patients]. Der Anaesthesist 2011;60:661-73.

8. Schuetz P, Albrich W, Mueller B. Procalcitonin for diagnosis of infection and guide to antibiotic decisions: past, present and future. BMC medicine 2011;9:107.

9. Nobre V, Harbarth S, Graf JD, Rohner P, Pugin J. Use of procalcitonin to shorten antibiotic treatment duration in septic patients: a randomized trial. American journal of respiratory and critical care medicine 2008;177:498-505.

10. Schuetz P, Chiappa V, Briel M, Greenwald JL. Procalcitonin algorithms for antibiotic therapy decisions: a systematic review of randomized controlled trials and recommendations for clinical algorithms. Archives of internal medicine 2011;171:1322-31.

11. Tang H, Huang T, Jing J, Shen H, Cui W. Effect of procalcitonin-guided treatment in patients with infections: a systematic review and meta-analysis. Infection 2009;37: 497-507.

12. Saeed K, Dryden M, Bourne S, Paget C, Proud A. Reduction in antibiotic use through procalcitonin testing in patients in the medical admission unit or intensive care unit with suspicion of infection. The Journal of hospital infection 2011;78:289-92.

13. Heyland DK, Johnson AP, Reynolds SC, Muscedere J. Procalcitonin for reduced antibiotic exposure in the critical care setting: a systematic review and an economic evaluation. Critical care medicine 2011;39:1792-9.

14. Wilke M, Grube RF, Bodmann KF. Guideline-adherent initial intravenous antibiotic therapy for hospital-acquired/ventilator-associated pneumonia is clinically superior, saves lives and is cheaper than non guideline adherent therapy. European journal of medical research 2011;16:315-23.

15. Wilke MH. Multiresistant bacteria and current therapy the economical side of the story. European journal of medical research 2010;15:571-6.

16. Moher D, Cook DJ, Eastwood S, Olkin I, Rennie D, Stroup DF. Improving the quality of reports of metaanalyses of randomised controlled trials: the QUOROM statement. QUOROM Group. The British journal of surgery 2000;87:1448-54.
17. Bouadma L, Luyt CE, Tubach F, et al. Use of procalcitonin to reduce patients' exposure to antibiotics in intensive care units (PRORATA trial): a multicentre randomised controlled trial. Lancet 2010;375:463-74.

18. Hochreiter M, Kohler T, Schweiger AM, et al. Procalcitonin to guide duration of antibiotic therapy in intensive care patients: a randomized prospective controlled trial. Critical care (London, England) 2009;13:R83.

19. Stolz D, Smyrnios N, Eggimann P, et al. Procalcitonin for reduced antibiotic exposure in ventilator-associated pneumonia: a randomised study. The European respiratory journal : official journal of the European Society for Clinical Respiratory Physiology 2009;34:1364-75.

20. Schroeder S, Hochreiter M, Koehler T, et al. Procalcitonin (PCT)-guided algorithm reduces length of antibiotic treatment in surgical intensive care patients with severe sepsis: results of a prospective randomized study. Langenbeck's archives of surgery / Deutsche Gesellschaft fur Chirurgie 2009;394:221-6.

21. Svoboda P, Kantorova I, Scheer P, Radvanova J, Radvan M. Can procalcitonin help us in timing of re-intervention in septic patients after multiple trauma or major surgery? Hepato-gastroenterology 2007;54:359-63.

22. Barbieri S, Feltracco P, Michieletto E, Basso I, Spagna A, Giron G. Demand and availability of Intensive Care beds. A study based on the data collected at the SUEM 118 Central of Padua from October 1996 to December 2001. Minerva anestesiologica 2003;69:625-34, 34-9.

23. Lin F, Chaboyer W, Wallis M. A literature review of organisational, individual and teamwork factors contributing to the ICU discharge process. Australian critical care : official journal of the Confederation of Australian Critical Care Nurses 2009;22:29-43.

24. Sidlow R, Aggarwal V. "The MICU is full": one hospital's experience with an overflow triage policy. Joint Commission journal on quality and patient safety / Joint Commission Resources 2011;37:456-60.

Received: July 8, 2011/ Accepted: November 10, 2011

Address for correspondence:

Dr. Michael H. Wilke

Dr. Wilke GmbH inspiring.health

Joseph-Wild-Str. 13,

D-81829 München

Germany

Tel.: $\quad+49(0) 8918908376-1$

Fax: $\quad+49(0) 8918908376-9$

E-mail: michael.wilke@d-w-g.de 